\title{
Relationship between Parotid Amylase Secretion and Osmolality in the Gastric Contents of Rats Fed a Pelleted or Liquid Diet
}

\author{
Masashi KURAHASHI and Koshiro INOMATA* \\ Department of Medical Sciences, School of Nursing and Social Services, and \\ *Department of Oral Physiology, Health Sciences University of Hokkaido, \\ Ishikari-Tobetsu, Hokkaido, 061-0293 Japan
}

\begin{abstract}
The relationship between parotid amylase secretion and the osmolality in the gastric contents of rats fed a pelleted or liquid diet was investigated. In sham-operated rats fed a pelleted diet, amylase activity in the parotid glands decreased, amylase activity in the plasma increased, and there was strong amylase activity in the gastric contents. As a result, both reducing sugar concentration and osmolality in the gastric contents increased. In parotid duct-ligated rats, the feeding of a pelleted diet affected neither parotid nor plasma amylase activity and there was little amylase activity in the gastric contents; this resulted in decreased starch digestion. The amylase activity in the gastric contents of rats fed a liquid diet was lower than that of rats fed the
\end{abstract}

pelleted diet. Both the reducing sugar concentration and osmolality in the gastric contents of rats fed the liquid diet were lower than those of rats fed the pelleted diet. However, both the reducing sugar concentration and osmolality in the gastric contents of rats fed the liquid diet were higher than those in the liquid diet itself. A small quantity of parotid amylase seems to effectively digest a large part of the starch in the stomaches of rats fed the liquid diet. These findings suggest that amylase secreted from parotid glands increases osmolality in the gastric contents via the production of reducing sugars from starch in rats when fed either pelleted or liquid diets. [Japanese Journal of Physiology, 49, 507-512, 1999]

Key words: liquid diet, parotid amylase, starch digestion, reducing sugars, gastric osmolality.

\begin{abstract}
Amylase activity decreases in the parotid glands of rats after feeding pelleted diet, while it appears in the gastric content [1]. Amylase is secreted from the parotid glands, moves from the oral cavity to the stomach and digests about one-third of the ingested starch in the stomach $[2,3]$. Reducing sugars such as maltose, maltotriose and $\alpha$-limit dextrins produced from the ingested starch by the parotid amylase are soluble in the water derived from diet, saliva, and gastric juice. Since the products of starch hydrolysis by parotid amylase are not absorbed in the stomach, the osmolality in the gastric contents is considered to increase with the production of reducing sugars.

The amylase activity in the parotid glands of rats remains unchanged after feeding a liquid diet, sug-
\end{abstract}

gesting that amylase secretion from parotid glands does not increase with a liquid diet [4-6]. However, amylase secretion from the parotid glands is not directly measured, and starch digestion in the stomach when feeding a liquid diet remains unknown.

To clarify the relationship between parotid amylase secretion and osmolality in the gastric contents of rats fed a pelleted or liquid diet, this study investigated the effects of both parotid duct ligation and feeding of a liquid diet on amylase secretion from parotid glands and starch digestion in the stomach in rats. The amylase activity in the gastric contents was used as the criterion for parotid amylase secretion in addition to the degree of depletion of gland amylase activity and increase in plasma amylase activity. The reducing

Received on April 28, 1999; accepted on September 27, 1999

Correspondence should be addressed to: Masashi Kurahashi, Department of Medical Sciences, School of Nursing and Social Services, Health Sciences University of Hokkaido, Ishikari-Tobetsu, Hokkaido, 061-0293 Japan. Tel: +81-1332-3-1499, Fax: +81-1332-3-1499, Email: kurahasi@hoku-iryo-u.ac.jp 
sugar concentration in the gastric contents was used as the criterion for starch digestion by parotid amylase in the stomach.

\section{MATERIALS AND METHODS}

Animals. All animal protocols followed the Guiding Principles for the Care and Use of Animals approved by the Council of the Physiological Society of Japan. Male Wistar rats, 6 weeks old and weighing 160-180 g, and 8 weeks old and weighing 240-280 g (Shizuoka Laboratory Animal Center), were kept in individual metabolic cages in an air-conditioned room $\left(22 \pm 2{ }^{\circ} \mathrm{C}\right.$, light on from 8 A.M. to 8 P.M.) and fed a commercial pelleted diet (Oriental MF, Oriental Yeast) and water ad libitum for 2 weeks prior to use.

In the first experiment, at 8 weeks of age, the rats were divided into two groups: a sham-operated group and a parotid duct-ligated group. Both parotid ducts were ligated $1 \mathrm{~cm}$ from the oral cavity with fine silk thread under anesthesia with sodium pentobarbital $(50 \mathrm{mg} / \mathrm{kg}$, intraperitoneally, Abbott Laboratories). The sham-operated rats were anesthetized with sodium pentobarbital, and the parotid ducts were exposed. To obtain a constant food intake during the feeding experiment, periodic food restrictions were imposed 2 weeks after the operation for both the parotid duct-ligated and sham-operated control rats. For a period of $10 \mathrm{~d}$, access to pelleted diet was limited to $4 \mathrm{~h}$ per day, from 10 A.M. to 2 P.M., while water was given ad libitum.

In the second experiment, at 10 weeks of age, the rats were divided into two groups. One group was fed a standard pelleted diet, while the other group was fed a liquid diet prepared daily by mixing two parts (wt) of water with one part (wt) of a powdered form of the standard diet (Oriental MF powdered, Oriental Yeast). To obtain a constant food intake during the feeding experiment, periodic food restrictions were imposed on both groups by limiting the free food access for a period of $10 \mathrm{~d}$ to $4 \mathrm{~h}$ per day, from 10 A.M. to 2 P.M., while water was given ad libitum.

Feeding experiment. On the 11th day after the start of the periodic food restrictions in both the first and second experiments, half of each group were kept unfed, while the other half was allowed to eat their regular pelleted or liquid diet for $1 \mathrm{~h}$. The amount of diet and water consumed, and the weight gains were noted. The rats fed or unfed were all sacrificed by cervical dislocation and bled $2 \mathrm{~h}$ after the start of feeding. Plasma was obtained from trunk blood by centrifugation and used for the assay of amylase activity.

Both parotid glands and stomach were quickly re- moved. The parotid glands were rinsed with ice-cold $0.9 \%$ saline, weighed, and homogenized with ice-cold $0.02 \mathrm{M}$ phosphate buffer $(\mathrm{pH} 7.0$ ) containing $0.05 \mathrm{M}$ $\mathrm{NaCl}$ in a Potter-Elvehjem glass homogenizer with a Teflon pestle. The homogenate was centrifuged at $2,000 \times g$ for $20 \mathrm{~min}$ at $4^{\circ} \mathrm{C}$, and the supernatant was used for the assay of amylase activity.

The stomach was dissected, and the gastric content was removed and weighed. The gastric content was cooled on ice, homogenized for $2 \mathrm{~min}$, and divided into two parts. The procedure on ice inhibited the additional production of reducing sugars from starch by parotid amylase. One part of the gastric content was heated for $15-18 \mathrm{~h}$ at $110^{\circ} \mathrm{C}$, and the water content was calculated by subtracting the weight after heating from that before heating. The other part of the gastric content was centrifuged at $2,000 \times g$ for $20 \mathrm{~min}$ at $4^{\circ} \mathrm{C}$. The supernatant was used for the assay of $\mathrm{pH}$, amylase activity, reducing sugar, sodium, potassium and chloride concentrations, and osmolality.

Assays. The amylase activity of plasma, parotid glands, and gastric contents was determined by the blue starch method of Ceska et al. [7] (Neo-Amylase Test, Daiichi Pure Chemical). Samples of plasma, parotid glands, and gastric contents were diluted 10 8,000 times before processing. The amylase activity was measured at $37^{\circ} \mathrm{C}$ and $\mathrm{pH}$ 7.0. The reducing sugars in the reaction mixture (maximum $1 \mathrm{mM}$ ) did not affect the amylase activity. The $\mathrm{pH}$ of the gastric content was measured at $4^{\circ} \mathrm{C}$. The reducing sugar concentration of the gastric contents was determined with 3,6-dinitrophthalic acid used as the color-developing agent [8]. The sodium and potassium concentrations of the gastric contents were measured with flame photometry (775-A, Hitachi). The chloride concentration of the gastric contents was determined using a coulometric titrator (CL-7, Hiranuma Sangyo). The osmolality of the gastric contents was determined by freezing point depression (Fiske $\mathrm{OM}^{\mathrm{TM}}$ Osmometer).

Statistics. Two-way analysis of variance (ANOVA) was performed on the data of the amylase activity of plasma and parotid glands. Post hoc individual comparisons were made with the Scheffe's $t$ test. Student's $t$ - or Welch's $t$-test was used to analyze differences between two groups.

\section{RESULTS}

\section{Body weight, and food and water intake}

Neither parotid duct ligation nor feeding of the liquid diet affected the body weight at fasting after $10 \mathrm{~d}$ of restricted feeding. The food and water intake during the $1 \mathrm{~h}$ of feeding was similar for the parotid duct- 
Table 1. Body weight before the $1 \mathrm{~h}$ feeding, and food and water intake during the $1 \mathrm{~h}$ of feeding.

\begin{tabular}{lccc}
\hline & $\begin{array}{c}\text { Body weight } \\
(\mathrm{g})\end{array}$ & $\begin{array}{c}\text { Food intake } \\
(\mathrm{g} / \mathrm{h})\end{array}$ & $\begin{array}{c}\text { Water intake } \\
(\mathrm{g} / \mathrm{h})\end{array}$ \\
\hline Experiment 1 & & & \\
Sham-operated & $281 \pm 5$ & $8.16 \pm 0.40$ & $6.9 \pm 0.5$ \\
Parotid duct-ligated & $294 \pm 6$ & $7.20 \pm 0.48$ & $8.1 \pm 0.9$ \\
Experiment 2 & & & \\
Pelleted diet & $291 \pm 5$ & $7.30 \pm 0.28$ & $5.5 \pm 0.5$ \\
Liquid diet & $300 \pm 4$ & $8.71 \pm 033^{*, \dagger}$ & $17.4 \pm 0.7^{\star *, \dagger}$
\end{tabular}

Values are means \pm SE of $8-9$ rats. ${ }^{*}$ Weight of powdered diet eaten. ${ }^{* *}$ Includes excess water with the liquid diet. ${ }^{\dagger} p<0.01$ vs. pelleted diet.
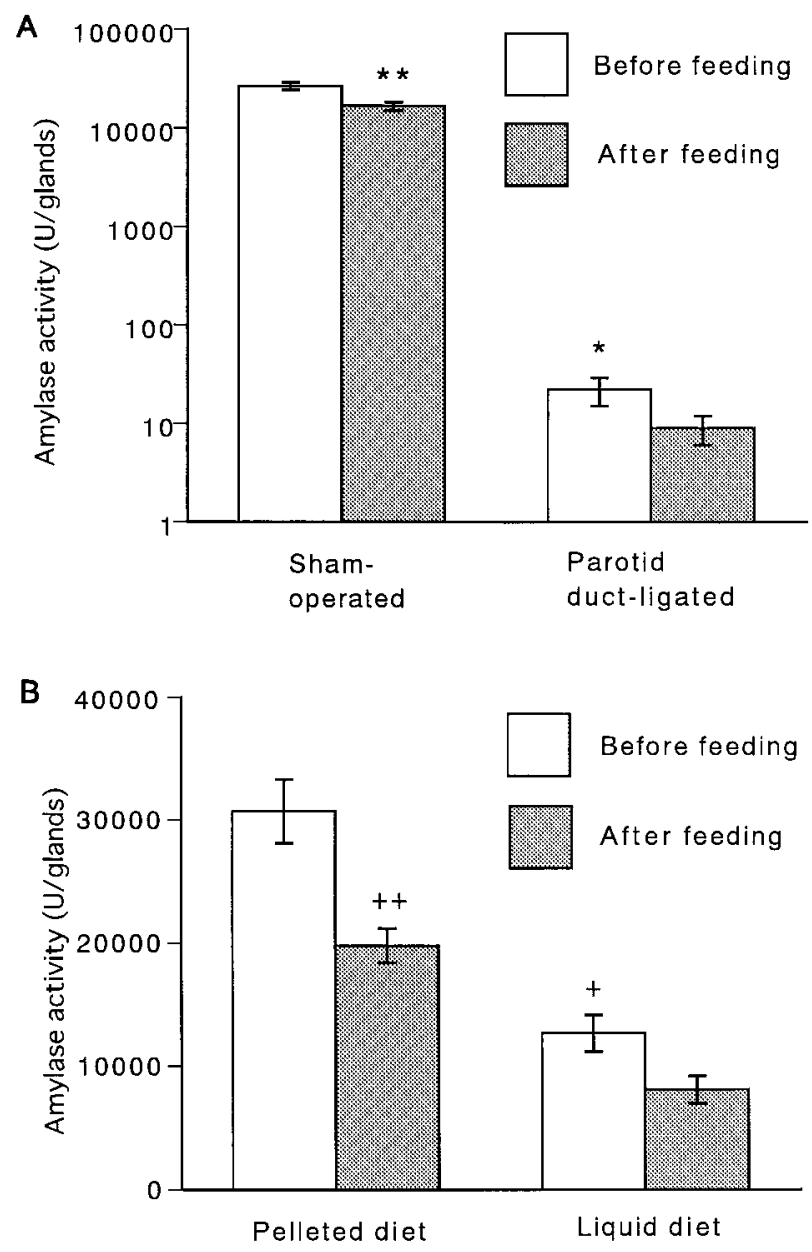

Fig. 1. Parotid amylase activity of sham-operated controls $(n=9$ or 10$)$ and parotid duct-ligated rats $(n=8$ or 9) before and after the $1 \mathrm{~h}$ feeding with pelleted diet (A), and the parotid amylase activity of rats before and after the $1 \mathrm{~h}$ feeding with pelleted ( $n=7$ or 8 ) or liquid diets $(\boldsymbol{n}=\mathbf{7}$ or $\mathbf{8}) \mathbf{( B )}$. Values are means \pm SE. ${ }^{*} p<0.001$ vs. sham-operated control, ${ }^{+} p<0.001$ vs. pelleted diet, ${ }^{* \star}$ and ${ }^{++} p<0.001$ vs. before feeding.

ligated and sham-operated control rats. The food intake during the $1 \mathrm{~h}$ of feeding was significantly higher
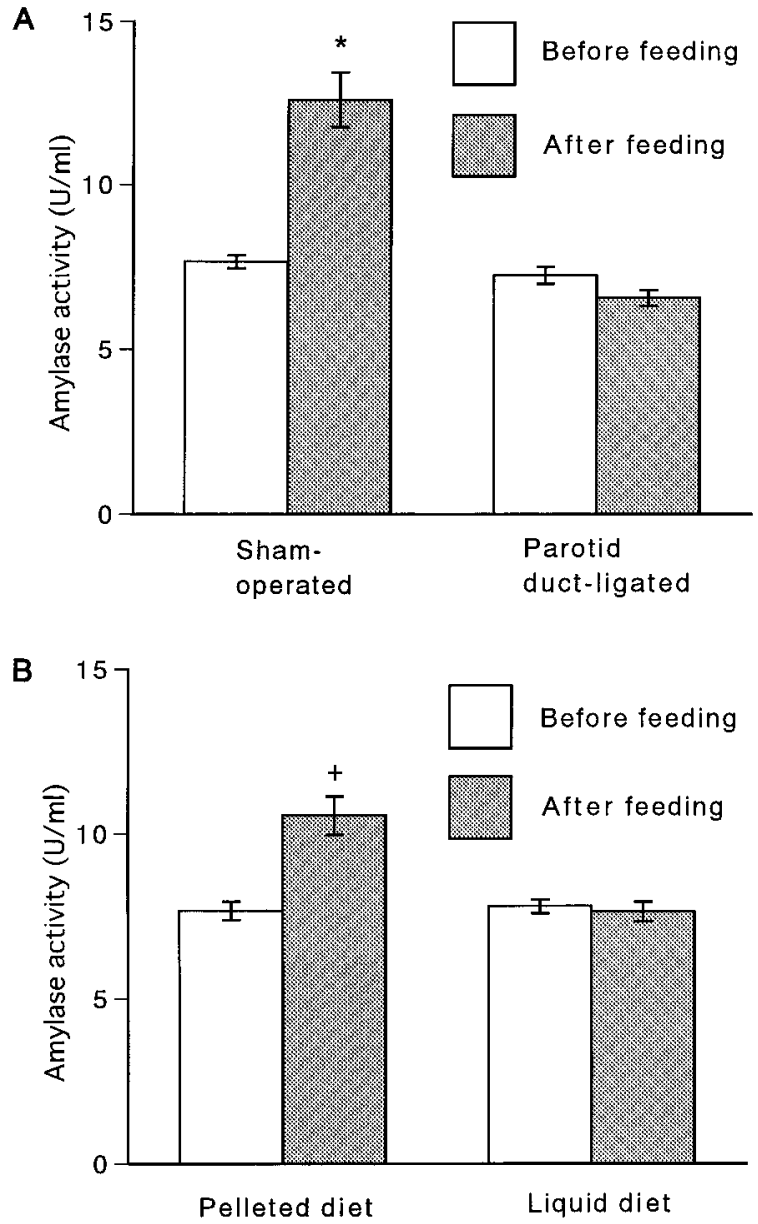

Fig. 2. Plasma amylase activity of sham-operated controls $(n=9$ or 10$)$ and parotid duct-ligated rats $(n=8$ or 9 ) before and after the $1 \mathrm{~h}$ feeding with pelleted diet (A), and the plasma amylase activity of rats before and after the $1 \mathrm{~h}$ feeding with pelleted ( $n=7$ or 8 ) or liquid diets $(\boldsymbol{n}=\mathbf{7}$ or $\mathbf{8})(\mathbf{B})$. Values are means \pm SE. * and ${ }^{+} p<0.001$ vs. before feeding.

in the rats fed the liquid diet than in the rats fed the pelleted diet (Table 1).

\section{Amylase activity in parotid glands}

In the sham-operated control rats, the amylase activity in the parotid glands decreased significantly after the $1 \mathrm{~h}$ feeding. The amylase activity in the parotid glands of the parotid duct-ligated rats was markedly lower than that of the sham-operated control rats at fasting, and feeding did not affect the amylase activity in the parotid glands of the parotid duct-ligated rats (Fig. 1A). The amylase activity in the parotid glands of the rats fed the liquid diet at fasting was markedly lower than that of the rats fed the pelleted diet, and feeding tended to decrease the amylase activity in the parotid glands of the rats fed the liquid diet, but the decrease in parotid amylase activity was not statistically significant. (Fig. 1B). 
Table 2. Weight, water content, $\mathrm{pH}$, and amylase activity in gastric contents after the $1 \mathrm{~h}$ of feeding.

\begin{tabular}{|c|c|c|c|c|}
\hline & $\begin{array}{l}\text { Weight } \\
\text { (g) }\end{array}$ & $\begin{array}{c}\text { Water content } \\
(\%)\end{array}$ & $\mathrm{pH}$ & $\begin{array}{c}\text { Amylase activity } \\
(\mathrm{U} / \mathrm{ml})\end{array}$ \\
\hline \multicolumn{5}{|l|}{ Experiment 1} \\
\hline Sham-operated & $11.04 \pm 0.78$ & $58.3 \pm 0.4$ & $5.95 \pm 0.04$ & $821 \pm 101$ \\
\hline Parotid duct-ligated & $9.37 \pm 0.72$ & $59.8 \pm 1.1$ & $5.76 \pm 0.05^{\star}$ & $0.16 \pm 0.01^{\dagger}$ \\
\hline \multicolumn{5}{|l|}{ Experiment 2} \\
\hline Pelleted diet & $8.61 \pm 0.58$ & $57.3 \pm 0.3$ & $5.95 \pm 0.04$ & $962 \pm 84$ \\
\hline Liquid diet & $13.72 \pm 0.94^{\star \star}$ & $71.2 \pm 0.2^{\star \star}$ & $\begin{array}{c}5.92 \pm 0.04 \\
(6.32 \pm 0.02)\end{array}$ & $6.8 \pm 3.2^{\star \star}$ \\
\hline
\end{tabular}

Values are means \pm SE of $8-9$ rats. ${ }^{*} p<0.01,{ }^{\dagger} p<0.001$ vs. sham-operated. ${ }^{* \star} p<0.001$ vs. pelleted diet. Value in parenthesis is the mean \pm SE of 4 samples of liquid diet.

Table 3. Reducing sugar, sodium, potassium and chloride concentrations, and osmolality in gastric contents after the $1 \mathrm{~h}$ of feeding.

\begin{tabular}{lcccccc}
\hline & $\begin{array}{c}\text { Reducing sugar } \\
(\mathrm{mM})\end{array}$ & $\begin{array}{c}{\left[\mathrm{Na}^{+}\right]} \\
(\mathrm{meq} / \mathrm{l})\end{array}$ & $\begin{array}{c}{\left[\mathrm{K}^{+}\right]} \\
(\mathrm{meq} / \mathrm{l})\end{array}$ & $\begin{array}{c}{\left[\mathrm{Cl}^{-}\right]} \\
(\mathrm{meq} / \mathrm{l})\end{array}$ & $\begin{array}{c}\text { Osmolality } \\
(\mathrm{mOsm} / \mathrm{kg})\end{array}$ & $\begin{array}{c}\text { Reducing sugar } \\
\text { Osmolality }\end{array}$ \\
\hline Experiment 1 & & & & & & \\
$\quad$ Sham-operated & $248 \pm 6$ & $92 \pm 3$ & $138 \pm 2$ & $154 \pm 5$ & $837 \pm 11$ & $29.7 \pm 0.6$ \\
$\quad$ Parotid duct-ligated & $41 \pm 7^{\dagger}$ & $61 \pm 2^{\dagger}$ & $136 \pm 4$ & $134 \pm 5^{\star}$ & $610 \pm 25^{\dagger}$ & $6.6 \pm 0.9^{\dagger}$ \\
Experiment 2 & & & & & \\
$\quad$ Pelleted diet & $277 \pm 6$ & $97 \pm 2$ & $136 \pm 1$ & $137 \pm 3$ & $870 \pm 14$ & $31.8 \pm 0.3$ \\
$\quad$ Liquid diet & $86 \pm 4^{\star *}$ & $38 \pm 1^{\star *}$ & $73 \pm 1^{\star *}$ & $57 \pm 1^{\star *}$ & $386 \pm 5^{\star *}$ & $22.3 \pm 0.9^{\star *}$ \\
& $(2 \pm 0)$ & $(34 \pm 0)$ & $(77 \pm 0)$ & $(41 \pm 1)$ & $(310 \pm 3)$ & $(0.7 \pm 0.0)$
\end{tabular}

Values are means \pm SE of $8-9$ rats. ${ }^{\star} p<0.02,{ }^{\dagger} p<0.001$ vs. sham-operated. ${ }^{\star \star} p<0.001$ vs. pelleted diet. Values in parentheses are means \pm SE of 4 samples of liquid diet.

\section{Amylase activity in plasma}

In the sham-operated control rats, the amylase activity in plasma increased significantly after the $1 \mathrm{~h}$ feeding. The amylase activity in the plasma of the parotid duct-ligated rats was not different from that of the sham-operated control rats at fasting, and feeding did not affect the amylase activity in the plasma of the parotid duct-ligated rats (Fig. 2A). The amylase activity in the plasma of the rats fed the liquid diet did not differ from that of the rats fed the pelleted diet at fasting; feeding did not affect the amylase activity in the plasma of the rats fed the liquid diet (Fig. 2B).

\section{Weight, water content, $\mathrm{pH}$, and amylase activ- ity in the gastric contents}

There were no differences between the weight and water contents of the gastric contents of the sham-operated control and parotid duct-ligated rats. The $\mathrm{pH}$ of the gastric contents was significantly lower in the parotid duct-ligated rats than in the sham-operated control rats. The amylase activity in the gastric contents was markedly lower in the parotid duct-ligated rats than in the sham-operated control rats. Both the weight and water contents of the gastric contents were significantly higher in the rats fed the liquid diet than in the rats fed the pelleted diet. The $\mathrm{pH}$ of the gastric contents did not differ for the two groups. The amylase activity in the gastric contents was markedly lower in the rats fed the liquid diet than in the rats fed the pelleted diet (Table 2).

\section{Reducing sugar, sodium, potassium and chloride concentrations, and osmolality in the gastric contents}

Parotid duct ligation markedly reduced the reducing sugar, sodium and chloride concentrations, osmolality, and the ratio of reducing sugar concentration to osmolality in the gastric contents, while this treatment did not affect the potassium concentration in the gastric contents. The reducing sugar, sodium, potassium and chloride concentrations, osmolality, and the ratio of reducing sugar concentration to osmolality in the gastric contents were markedly lower in the rats fed the liquid diet than in the rats fed the pelleted diet (Table $3)$. 


\section{DISCUSSION}

Both the decrease in parotid amylase activity $[1,4-6$, $9,10]$ and increase in plasma amylase activity [11] are known to reflect the amylase secretion from parotid glands in normal rats when feeding a pelleted diet. At the moderately acidic $\mathrm{pH}$ in the rat stomach, the amylase secreted from the parotid glands maintains its activity $[2,3]$, similar to the situation in humans [12], and for normal rats fed a pelleted diet, the amylase activity in the gastric contents is considered to reflect the amylase secretion from the parotid glands.

In the sham-operated control rats, the amylase activity in the parotid glands decreased and that in the plasma increased when feeding the pelleted diet. The decrease in gland amylase activity during feeding was about 9,800 U/total glands, and there was strong amylase activity in the gastric contents (about 5,300 U/ total content); as a result, both the reducing sugar concentration and osmolality in the gastric contents increased. There was little amylase activity in the parotid glands of the parotid duct-ligated rats at fasting (about $22 \mathrm{U} /$ total glands), and the feeding of pelleted diet affected neither the parotid nor plasma amylase activity, indicating that amylase secretion from the parotid glands decreases markedly when feeding a pelleted diet, and that this results in decreased amylase activity in the gastric contents of the parotid duct-ligated rats (about $1 \mathrm{U} /$ total content). Both the reducing sugar concentration and osmolality in the gastric contents were lower in the parotid duct-ligated rats than in the sham-operated control rats. These findings suggest that parotid amylase increases osmolality in the gastric contents via the production of reducing sugars from starch when fed a pelleted diet.

About $2 \%$ of the parotid amylase activity occurs in von Ebner's glands and the amylase is secreted from von Ebner's glands when feeding a pelleted diet [13]. The small amount of amylase in the gastric contents of parotid duct-ligated rats fed a pelleted diet may derive from von Ebner's glands, and this may produce a small amount of reducing sugars in the gastric contents.

The lower $\mathrm{pH}$ of the gastric contents of the parotid duct-ligated rats suggests a reduced salivary bicarbonate secretion. Sodium and chloride concentrations in the gastric contents were significantly lower in the parotid duct-ligated rats than in the sham-operated control rats, suggesting that parotid salivary secretion is inhibited by the ligation of the parotid duct. The decrease in sodium chloride secretion from the parotid glands may explain some of the decrease in gastric osmolality in the parotid duct-ligated rats fed the pel- leted diet.

At fasting after $10 \mathrm{~d}$ of restricted feeding, the amylase activity in the parotid glands was markedly lower in the rats fed the liquid diet than in the rats fed the pelleted diet. These results agree with previous studies [14-16]. The $1 \mathrm{~h}$ feeding with a liquid diet affected neither parotid nor plasma amylase activity, and the amylase in the gastric contents was markedly lower in the rats fed the liquid diet than in the rats fed the pelleted diet. Both the reducing sugar concentration and osmolality in the gastric contents were lower in the rats fed the liquid diet than in the rats fed the pelleted diet. These findings suggest that the decrease in amylase secretion from the parotid glands results in a decrease in both reducing-sugar production from starch and osmolality in the gastric contents of the rats fed the liquid diet. The increase in water intake and water content of the gastric contents of rats fed the liquid diet indicates that the gastric contents were diluted with ingested water. The decreases in the sodium and chloride concentrations were significantly larger than the decrease in potassium concentration in the gastric contents of the rats fed the liquid diet, suggesting that parotid salivary secretion decreased in the rats fed the liquid diet. Both the decrease in parotid salivary secretion and dilution of gastric contents with ingested water explain the marked decrease in the osmolality of the gastric contents of the rats fed the liquid diet. In a preliminary study, it was observed that the gastric emptying of foods was faster in rats fed a liquid diet than in rats fed a pelleted diet. The increased gastric emptying of foods in the rats fed the liquid diet may decrease the amylase activity, reducing sugar concentration, and osmolality in the gastric contents.

The total amylase activity in the gastric contents of the rats fed the pelleted diet was $4,746 \mathrm{U}$, and it was $66.4 \mathrm{U}$ with the liquid diet. Although the amylase activity in the gastric contents was markedly lower in the rats fed the liquid diet than in the rats fed the pelleted diet, the reducing sugar concentration in the gastric contents of the rats fed the liquid diet was higher than that in the liquid diet itself. The reducing sugar produced in the gastric contents of the rats fed the pelleted diet for $2 \mathrm{~h}$ was $1.37 \mathrm{mmol}$, and it corresponds to $11.4 \mathrm{U}$ of amylase activity; this activity was $0.25 \%$ of the total amylase activity in the gastric contents. The reducing sugar produced in the gastric contents of the rats fed the liquid diet for $2 \mathrm{~h}$ was $0.84 \mathrm{mmol}$, corresponding to $7 \mathrm{U}$ of amylase activity, $10.5 \%$ of the total amylase activity in the gastric contents. A small quantity of parotid amylase seems to effectively digest a large part of the starch in the gastric contents of the rats fed the liquid diet. The physiological role of a 
small quantity of parotid amylase has been observed in the intestinal contents of exocrine pancreatic-insufficient rats [17]. The increased water content in the gastric contents of the rats fed the liquid diet may increase the interaction between amylase and its substrate. The osmolality in the liquid diet was similar to that of plasma [18], while the osmolality of the gastric contents of the rats fed the liquid diet became hypertonic. Amylase secreted from parotid glands increases the osmolality in the gastric contents via the production of reducing sugars from starch when feeding pelleted or liquid diets.

We are grateful to Professor T. Christensen, Hokusei Junior College, for proofreading this manuscript. This work was supported by a Grant-in-Aid for Scientific Research (M. Kurahashi; No. 088350200) from the Ministry of Education, Science, Sports and Culture of Japan.

\section{REFERENCES}

1. Kurahashi $\mathrm{M}$ and Inomata $\mathrm{K}$ : Amylase secretion by parotid glands and pancreas of diabetic rats during feeding. Am J Physiol 254: G878-G882, 1988

2. Kurahashi $\mathrm{M}$ and Inomata K: Role of parotid amylase in starch digestion in the gastro-intestinal tracts of diabetic rats. J Dent Res 68: 1366-1369, 1989

3. Kurahashi $M$ and Inomata $K$ : Starch digestion by parotid amylase in the gastrointestinal tract of rats with pancreatitis. Jpn J Oral Biol 38: 326-334, 1996

4. Asztély A, Tobin G, and Ekström J: Masticatory-salivary reflexes mobilize non-adrenergic, non-cholinergic secretory mechanisms in parotid glands of conscious rats. Acta Physiol Scand 151: 373-376, 1994

5. Johnson DA and Sreebny LM: Effect of food consistency and starvation on the diurnal cycle of the rat parotid gland. Arch Oral Biol 16: 177-185, 1971

6. Schneyer CA: Role of sympathetic pathway in secretory activity induced in rat parotid by feeding. Proc Soc Exp Biol Med 147: 316-317, 1974
7. Ceska M, Birath $K$, and Brown B: A new and rapid method for the clinical determination of $\alpha$-amylase activities in human serum and urine. Optimal conditions. Clin Chim Acta 26: 437-444, 1969

8. Momose T, Murai $Y$, and Watanabe M: Determination of reducing sugars with 3,6-dinitrophthalic acid. Talanta 5: 275-278, 1960

9. Harrop TJ and Garrett JR: Effects of preganglionic sympathectomy on secretory changes in parotid acinar cells of rats on eating. Cell Tissue Res 154: 135-150, 1974

10. Speirs RL and Hodgson C: Control of amylase secretion in the rat parotid gland during feeding. Arch Oral Biol 21: 539-544, 1976

11. Proctor GB, Asking B, and Garrett JR: Effects of secretory nerve stimulation on the movement of rat parotid amylase into the circulation. Arch Oral Biol 34: 609613, 1989

12. Rosenblum JL, Irwin CL, and Alpers DH: Starch and glucose oligosaccharides protect salivary-type amylase activity at acid pH. Am J Physiol 254: G775-G780, 1988

13. Hoshi M, Kurahashi M, Inomata K, Obara $N$, and Takeda M: Autonomic regulation of amylase secretion from von Ebner's glands in rats during feeding. Dentistry Jpn 30: 40-43, 1993

14. Hall HD and Schneyer CA: Salivary gland atrophy in rat induced by liquid diet. Proc Soc Exp Biol Med 25: 789-793, 1964

15. Hand AR and Ho B: Liquid-diet-induced alterations of rat parotid acinar cells studied by electron microscopy and enzyme cytochemistry. Arch Oral Biol 26: 369380, 1981

16. Sreebny LM and Johnson DA: Effect of food consistency and decreased food intake on rat parotid and pancreas. Am J Physiol 215: 455-460, 1968

17. Kurahashi $M$ and Inomata $K$ : Starch digestion by parotid amylase in the gastrointestinal tract of exocrine pancreatic insufficient rats. Jpn J Oral Biol 38: 335344, 1996

18. Kawabata T: Effect of hydration states on thermoregulatory responses during exercise in rats. Jpn $\mathrm{J}$ Physiol 48: 57-62, 1998 